\title{
Vulnérabilité des ménages et sentiment de déclassement social : l'adoption de comportements écologiques peut-elle contribuer à satisfaire les motivations identitaires dans un contexte territorialisé ?
}

Household vulnerability and feeling of social downgrading: can the adoption of ecological behaviors contribute to satisfying identity motivations in a territorialized context?

Sandrine Gombert-Courvoisier, Olivier Ballesta, Aurélie Carimentrand, Francis Ribeyre, Elsa Causse, Florian Delerue, Marie-Line Félonneau et Geoffrey Rioche

\section{(2) OpenEdition}

Édition électronique

URL : https://journals.openedition.org/popvuln/1082

DOI : 10.4000/popvuln. 1082

ISSN : 2650-7684

Éditeur

LIR3S - Laboratoire Interdisciplinaire de Recherche "Sociétés Sensibilités Soin" (UMR 7366 CNRS-uB)

Édition imprimée

Date de publication : 1 mars 2019

Pagination : $57-77$

ISBN : 978-2-918173-26-7

ISSN : 2269-0182

Référence électronique

Sandrine Gombert-Courvoisier, Olivier Ballesta, Aurélie Carimentrand, Francis Ribeyre, Elsa Causse, Florian Delerue, Marie-Line Félonneau et Geoffrey Rioche, « Vulnérabilité des ménages et sentiment de déclassement social : l'adoption de comportements écologiques peut-elle contribuer à satisfaire les motivations identitaires dans un contexte territorialisé ? », Populations vulnérables [En ligne], 5 | 2019, mis en ligne le 26 mai 2020, consulté le 26 février 2022. URL : http://journals.openedition.org/popvuln/ 1082 ; DOI : https://doi.org/10.4000/popvuln.1082

Ce document a été généré automatiquement le 26 février 2022.

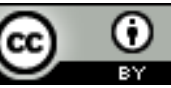

Les contenus de la revue Populations vulnérables sont mis à disposition selon les termes de la Licence Creative Commons Attribution 4.0 International 


\section{Vulnérabilité des ménages et sentiment de déclassement social : l'adoption de comportements écologiques peut-elle contribuer à satisfaire les motivations identitaires dans un contexte territorialisé ?}

Household vulnerability and feeling of social downgrading: can the adoption of ecological behaviors contribute to satisfying identity motivations in a territorialized context?

Sandrine Gombert-Courvoisier, Olivier Ballesta, Aurélie Carimentrand, Francis Ribeyre, Elsa Causse, Florian Delerue, Marie-Line Félonneau et Geoffrey Rioche

Cet article présente des résultats du projet de recherche Inove.com (Identité, Norme, Vulnérabilité, pour une approche écologique de la consommation durable des ménages), financé par le ministère de l'Environnement, de l'Énergie et de la Mer, dans le cadre du second appel à projet MOVIDA (2013-2016).

Les auteurs souhaitent remercier les répondants au questionnaire, les enquêteurs vacataires et les structures d'accueil ayant permis la passation du questionnaire en vis-à-vis, ainsi que l'ensemble des stagiaires ayant travaillé sur ces données. 


\section{Introduction}

1 En ce début de $\mathrm{xxI}^{\mathrm{e}}$ siècle, les évolutions sociétales récentes nous amènent à considérer que nous vivons une transition à la fois écologique (raréfaction des ressources et dégradation des milieux) mais également sociale et économique (augmentation des inégalités et détérioration du pouvoir d'achat). Ces évolutions ont comme conséquence une réorganisation des modes de vie et de consommation des ménages en lien avec leur qualité de vie. Une frange nouvelle de la population se retrouve, sous l'effet de perturbateurs, dans une situation de vulnérabilité, dès lors qu'elle se voit privée d'une partie des biens et services auxquels elle avait accès auparavant. Ces ménages risquent ainsi de se retrouver en situation de déclassement social avec pour conséquences des difficultés d'ordre psychologique, social, économique et sanitaire. Ces perturbateurs étant souvent territorialisés, les risques de vulnérabilité peuvent alors différer en fonction des territoires (Inove.com, 2016). Cette situation peut alors pousser les ménages concernés à adopter, de façon contrainte, des changements de comportements essentiellement pour des raisons économiques. Or, les comportements promouvant des valeurs de sobriété, d'évitement des gaspillages et de réduction de la consommation des ressources répondent autant à des motivations d'ordre économique que d'ordre environnemental. L'adoption de comportements écologiques faisant de plus en plus l'objet d'une valorisation sociale au travers d'une norme proenvironnementale (Félonneau et Becker, 2008), ces changements de pratiques pourraient alors représenter des supports d'estime personnelle et ainsi contribuer à atténuer le sentiment de déclassement social des personnes vulnérables (Ballesta et al., 2016).

2 De nombreux travaux ont été effectués en lien avec la vulnérabilité des populations et les inégalités environnementales (Pye et al., 2008 ; Laurent, 2010), sur l'écologisme des pauvres (Alier, 2014) ou encore sur les nouveaux modes de vie durables (Bourg et al., 2016). Cependant, peu de recherches visent à cerner les conséquences des changements comportementaux vers des pratiques plus durables vis-à-vis du bien-être des populations vulnérables, dans un contexte territorialisé. Plusieurs questions se posent alors. Tout d'abord, comment identifier des perturbateurs pouvant impacter la qualité de vie des personnes ? Les perturbateurs et le sentiment de déclassement social sont-ils perçus différemment en fonction des territoires? L'adoption de comportements écologiques contribue-t-elle à une augmentation de la satisfaction des motivations identitaires permettant ainsi d'atténuer le sentiment de déclassement social et d'améliorer la qualité de vie des personnes vulnérables?

\section{II. Éléments de définition et cadrage théorique}

\section{1) Le sentiment de déclassement social}

Dans cet article, nous avons retenu une approche subjective du déclassement social. La notion de déclassement peut en effet s'appréhender en fonction d'indicateurs objectifs (par exemple une diminution de revenus), mais elle peut également renvoyer à des éléments subjectifs tels que des perceptions ou des craintes exprimées individuellement ou collectivement (Boisson, 2009). La hantise du déclassement, qui repose sur la croyance que chacun risque à tout moment de perdre son emploi, son salaire, ses privilèges ou son statut, en est un bon exemple (Maurin, 2009). Par exemple, 
on peut parfaitement imaginer qu'une personne qui vit objectivement une situation de mobilité sociale descendante à laquelle elle peut s'adapter n'en est pas affectée psychologiquement et ne s'estime pas déclassée alors qu'une autre personne, dans la même situation, peut vivre le déclassement social à la fois aux plans objectif et subjectif. C'est donc à la fois la situation objective et la perception de cette situation qui définissent notre approche du déclassement social.

\section{2) Les perturbateurs de la qualité de vie} origines susceptibles de déstabiliser une société de façon relativement rapide et profonde, pouvant conduire à un changement structurel et fonctionnel peu réversible, généralement perçu et vécu de façon négative par la majorité de la population.

5 Ces perturbateurs peuvent alors agir comme des "déclencheurs" de changements comportementaux susceptibles de conduire à un sentiment de déclassement social chez certaines personnes vulnérables. Peuvent être distingués les perturbateurs « macro » à large échelle, applicables à l'ensemble de la population (comme par exemple l'évolution démographique ou la dégradation des milieux), des perturbateurs "micro» plus spécifiques à chaque ménage et conditionnant directement les pratiques de consommation associées à la qualité de vie. Pour cette recherche, nous nous intéressons plus particulièrement à ce deuxième type de perturbateurs.

6 Nous partons de l'hypothèse que les perturbateurs entrainnant le déclassement social peuvent être contrastés en fonction des territoires, en particulier selon le degré d'urbanisation. Par exemple, les transformations du tissu urbain de certaines villes centres (le cœur des métropoles en particulier) et les recompositions sociales qui les accompagnent sont potentiellement de puissants éléments perturbateurs. La réhabilitation des logements anciens et les opérations de requalification urbaine renforcent l'attractivité qu'exercent certains quartiers centraux auprès de classes sociales aisées. Ce processus de gentrification provoque une hausse des prix de l'immobilier qui limite l'installation des ménages des classes moyennes et populaires ou oblige certains résidents à déménager en banlieue. Cette évolution subie du cadre de vie peut ainsi être vécue comme un signe de déclassement. Par ailleurs, le processus de périurbanisation pousse de nombreuses familles à s'éloigner de plus en plus de leur lieu de travail qui reste concentré dans les grands pôles urbains. Or, le renchérissement du prix des carburants, l'accroissement des temps de déplacement et l'adoption de nouvelles normes en matière de circulation sont amenés à jouer un rôle perturbateur dans le quotidien des individus qui habitent ces territoires. La question se pose avec une acuité toute particulière pour les ménages dont l'équilibre financier est fragile et qui se sont parfois lourdement endettés pour accéder à la propriété. Enfin, certains territoires ruraux sont aujourd'hui confrontés à un processus de marginalisation lié en grande partie au délitement de la sphère productive et à l'éloignement des pôles urbains les plus dynamiques. Les populations résidentes (personnes sans emploi, personnes âgées) vivent quotidiennement les problèmes d'accès à certains services de base, en particulier dans le domaine sanitaire, et peuvent subir ou ressentir un déclassement social particulièrement fort. 


\section{3) Les comportements écologiques}

7 Les comportements écologiques sont généralement considérés comme des modes de consommation qui favorisent la réduction des impacts environnementaux. Nous avons choisi d'élargir et de complexifier cette définition strictement environnementale des comportements en y associant les dimensions sanitaires (meilleurs pour la santé), sociales (favorisant le lien social), économiques (moins chers à mettre en œuvre) et culturelles (pratiques historiques, éducation). Ces comportements écologiques sont très nombreux et diffèrent selon les postes de consommation (alimentation, transport, etc.) ou les thématiques liées à la consommation (énergie, eau, déchet, etc.). Nous avons donc choisi de les catégoriser en cinq types de pratiques opérationnelles: l'autoproduction liée au "faire soi-même"; la prolongation de la durée de vie des produits; la mutualisation de biens et de services; la réduction d'achats voire de consommation au sens large; et la substitution de produits ou de comportements par des produits, comportements ou services plus vertueux. Ces pratiques peuvent être reliées aux différents leviers préconisés par la Stratégie nationale de transition écologique vers un développement durable (SNTEDD 2015-2020) pour «réorienter les modèles de production, d'échange et de consommation » et «aider à la modification des choix et des comportements de la société ».

8 D'autres types de motivations peuvent être associés à l'adoption ou au maintien de ces nouvelles pratiques : les motivations identitaires.

\section{4) Les motivations identitaires}

9 Parmi les différentes approches théoriques de l'identité, nous avons retenu l'approche de Vignoles et al. (2006) qui permet d'identifier six motivations conceptuellement distinctes pouvant être impliquées dans les processus de construction de l'identité. Ce modèle considère donc l'identité comme un processus basé sur un ensemble de motivations différentes : les individus sont motivés à se voir positivement (estime de soi); croire que leurs identités sont continues dans le temps malgré les changements significatifs de la vie et qu'elles ne changent pas selon le lieu ou le contexte (continuité) ; se différencier des autres personnes (distinctivité); penser que leur vie est significative (sens); croire qu'ils sont compétents et capables d'influencer leur environnement (efficacité) ; ressentir qu'ils sont inclus et acceptés par les autres (appartenance).

10 Nous faisons l'hypothèse que plusieurs motivations identitaires pourraient ainsi influencer les comportements écologiques. La motivation d'efficacité, par exemple, peut jouer un rôle pivot entre le raisonnement et le passage à l'action : c'est parce que les personnes croient en l'efficacité de leurs actes qu'elles peuvent transformer leurs pensées en conduites. De plus, le constat de la réussite des comportements d'autres individus auxquels elles peuvent se référer renforce la motivation, permet de préciser les attentes de résultats de l'action et de percevoir les obstacles plus clairement.

\section{Matériels et méthodes}

11 Afin d'apporter des éléments de réponse quant aux interactions entre territoires, perturbateurs et déclassement social, trois zones d'études, situées le long d'un gradient de métropolisation, ont été retenues. Il s'agit de trois établissements publics de 
coopération intercommunale (EPCI): Bordeaux Métropole, la communauté de communes du Pays Créonnais et celle du Pays Foyen (Figure 1).

Figure 1. Localisation des trois territoires d'étude

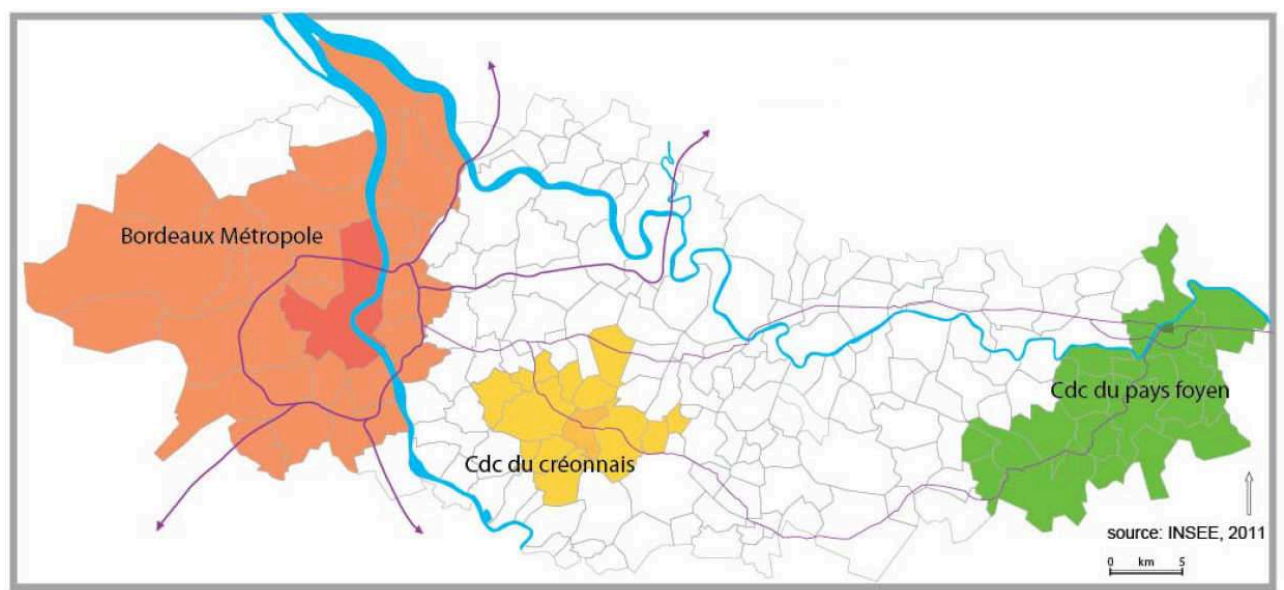

Une étude comparative a été réalisée en mobilisant de nombreux indicateurs sociodémographiques et économiques empruntés aux bases de données publiques (INSEE). Le but était d'élaborer un diagnostic cartographique faisant émerger les caractéristiques principales de chaque territoire et de mettre en exergue certains perturbateurs. Par ailleurs, un recensement des structures locales d'accompagnement dans le champ social et environnemental (centres d'animation socio-culturels) ou en lien avec les pratiques de consommation durable (recycleries) a également été effectué sur chacun de ces territoires.

13 La méthode de recueil d'informations correspond à une enquête par questionnaire. $\mathrm{Ce}$ questionnaire basé sur les méthodes de la psychologie sociale a été administré auprès de 285 répondants répartis équitablement sur les trois territoires d'étude ( $\mathrm{n}=98$ sur Bordeaux Métropole ; $\mathrm{n}=88$ sur la communauté de communes du Pays Créonnais ; $\mathrm{n}=99$ sur la communauté de communes du Pays Foyen). De façon à cibler des personnes en situation de déclassement potentiel, nous avons choisi d'effectuer la passation du questionnaire en vis-à-vis auprès des structures locales d'accompagnement des populations préalablement identifiées sur les territoires d'étude. Ce questionnaire est composé de thèmes qui traitent, entre autres, des perturbateurs craints ou vécus, du sentiment de déclassement social, des comportements écologiques mis en œuvre, des motivations identitaires ainsi que des leviers d'actions jugés pertinents pour tendre vers une consommation plus économe et plus écologique.

Afin de mettre en évidence l'influence de certains perturbateurs sur la qualité de vie des gens, deux questions ont été posées dans le questionnaire : «Considérez-vous que les événements suivants ${ }^{1}$ peuvent diminuer la qualité de vie des gens? »; "Êtes-vous, ou pourriezvous dans les 2 ans à venir être concerné(e) par ces événements?", les réponses étant cochées sur une échelle de $1:$ pas du tout à 6 : tout à fait.

Au sein de l'échantillon, l'identification des personnes en déclassement a été mesurée à l'aide d'une question («Comment définiriez-vous votre mode de vie?») pour laquelle étaient proposées deux échelles. Les répondants étaient amenés à placer un tiret vertical sur chaque échelle de même longueur («votre niveau de vie aujourd'hui »; « votre niveau de vie il y a quelques années »). Le sentiment de déclassement a été défini par la 
mesure de l'écart numérique (en $\mathrm{cm}$ ) entre les deux tirets verticaux. Pour un répondant indiquant une augmentation de son niveau de vie aujourd'hui par rapport à il y a quelques années (valeur positive de l'écart numérique), nous avons estimé qu'il ne ressentait pas de sentiment de déclassement. En revanche, pour un répondant indiquant que son niveau de vie lui paraissait inférieur aujourd'hui par rapport à il y a quelques années (valeur négative de l'écart numérique), nous avons estimé qu'il exprimait un sentiment de déclassement. Sur la base de l'écart numérique relatif à cette mesure, quatre classes ont été définies. Deux classes correspondant à des valeurs négatives: les personnes percevant une forte diminution de leur niveau de vie et caractérisées comme "très déclassées" (classe a: $n=73,-7,5<x<-1,5 \mathrm{~cm}$ ) et les personnes percevant une diminution moindre de leur niveau de vie et caractérisées comme « déclassées » (classe $\mathrm{b}: \mathrm{n}=73,-1,5<x<0 \mathrm{~cm}$ ). Deux autres classes correspondant à des valeurs positives : les personnes percevant une faible amélioration de leur niveau de vie et caractérisées comme "pas déclassées » (classe c: $n=66,0<x<0,9 \mathrm{~cm}$ ) et les personnes percevant une forte amélioration de leur niveau de vie et caractérisées comme " pas du tout déclassées » (classe $\mathrm{d}: \mathrm{n}=66,0,9<x<6,3 \mathrm{~cm}$ ).

Pour établir un lien éventuel entre la pratique des comportements écologiques et la satisfaction des motivations identitaires, les questions suivantes ont été posées (par exemple, ici, pour l'autoproduction) : «Le faites-vous? (sur une échelle de $1:$ jamais à 6: le plus souvent possible); "Produire ou faire des choses vous-même... ": "... vous distingue-t-il d'autres personnes? (distinctivité) ; « ... vous permet-il d'être la même personne, quels que soient le lieu, la situation ou le contexte?» (continuité); « ... vous donne-t-il une bonne image de vous-même?" (estime de soi); "... vous fait-il vous sentir compétent et capable? » (efficacité) ; "... donne-t-il du sens à votre vie ?» (sens); «... vous donne-t-il le sentiment d'appartenir à un groupe? » (appartenance), les réponses étant cochées sur une échelle de $1:$ pas du tout à $6:$ tout à fait.

17 Enfin, l'identification de leviers d'action a été mesurée par les deux questions suivantes : "Qu'est-ce qui facilite ou faciliterait ces comportements?"; "Qui vous aide ou pourrait vous aider à adopter ces comportements?».

Différentes analyses statistiques ont été effectuées pour mettre en évidence des liens entre les différents résultats et valider ou infirmer nos hypothèses de départ : ANOVA, test de Friedman, tests de corrélation de Spearman, test de comparaison de moyennes de Kruskall-Wallis.

\section{Résultats}

\section{1) Vulnérabilités territoriales et perturbateurs associés}

De fortes disparités socio-démographiques et économiques sont observées entre les territoires. À titre d'exemple, le territoire rural du Pays Foyen se distingue par l'importance relative des personnes de plus de 60 ans et par un taux de chômage particulièrement élevé, ce qui peut illustrer une certaine vulnérabilité sur ce territoire situé en marge du processus de métropolisation (Figure 2). Le territoire du Créonnais affiche depuis plusieurs décennies un grand dynamisme démographique principalement fondé sur son attractivité migratoire. De nombreux ménages issus des classes moyennes ont pu y satisfaire leur projet d'accession à la propriété individuelle, motivés par la recherche d'un "compromis géographique» entre coût du foncier, 
qualité de vie, et distance-temps vis-à-vis du pôle d'emploi bordelais. C'est sur ce territoire que la plus grande proportion de propriétaires est observée (Figure 3). La vulnérabilité des ménages habitant ce territoire périurbain semble alors davantage liée aux contraintes de la mobilité quotidienne qui découlent de la très forte dépendance vis-à-vis de Bordeaux en matière d'emplois. Enfin, le territoire de Bordeaux Métropole, de par son périmètre étendu, présente une grande hétérogénéité socio-spatiale et des dynamiques très contrastées, induisant une vulnérabilité plus ou moins importante des communes face à certains perturbateurs. Les communes situées sur la rive droite de la Garonne comptent parmi celles ayant le niveau de vie le plus faible, en lien avec un taux de chômage élevé. À l'inverse, les communes du nord-ouest montrent une surreprésentation de cadres et de professions intellectuelles supérieures (Figure 4).

Figure 2. Part des personnes âgées de plus de 60 ans selon l'unité territoriale (Bordeaux Métropole, communauté de communes du Créonnais, communauté de communes du Pays Foyen)

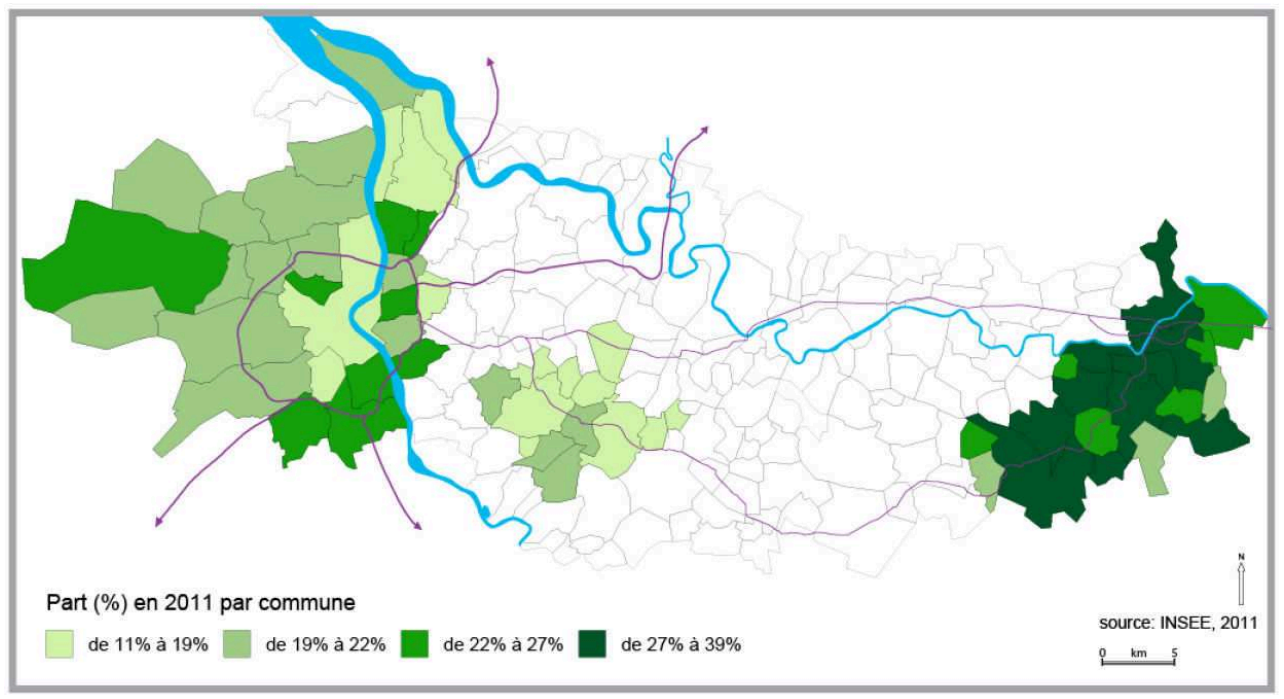

Figure 3. Part des résidences principales occupées par des propriétaires selon l'unité territoriale (Bordeaux Métropole, communauté de communes du Créonnais, communauté de communes du Pays Foyen)

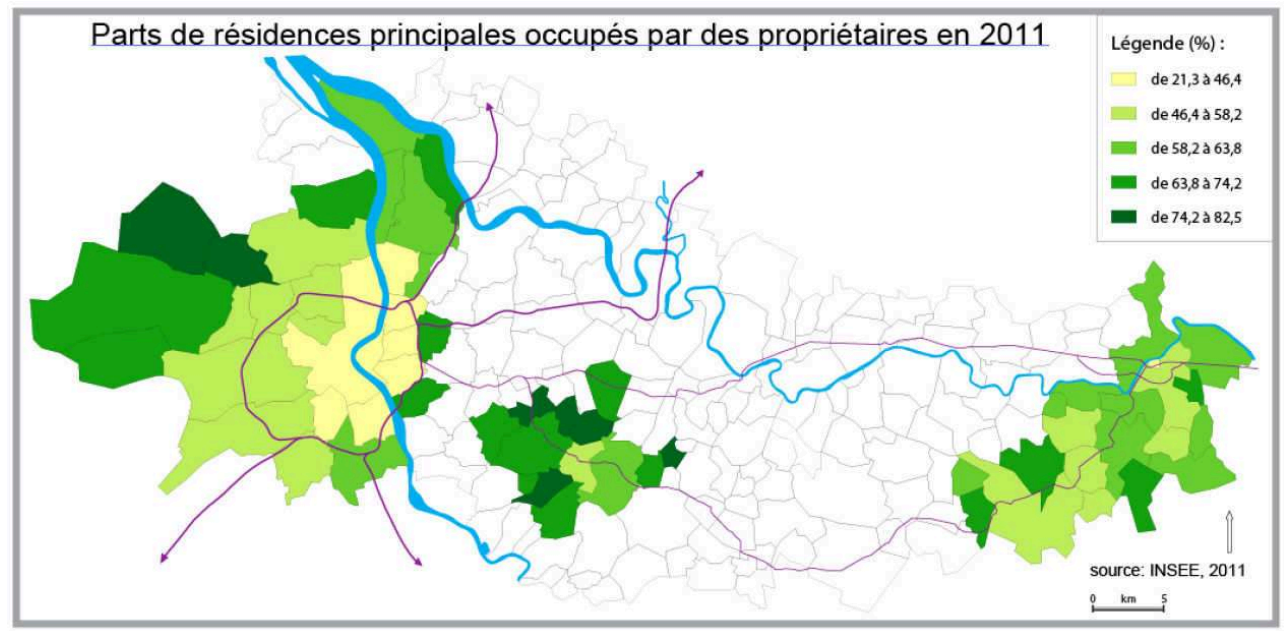

Populations vulnérables, 5 | 2019 
Figure 4 Revenu moyen des foyers fiscaux selon l'unité territoriale (Bordeaux Métropole, communauté de communes du Créonnais, communauté de communes du Pays Foyen)

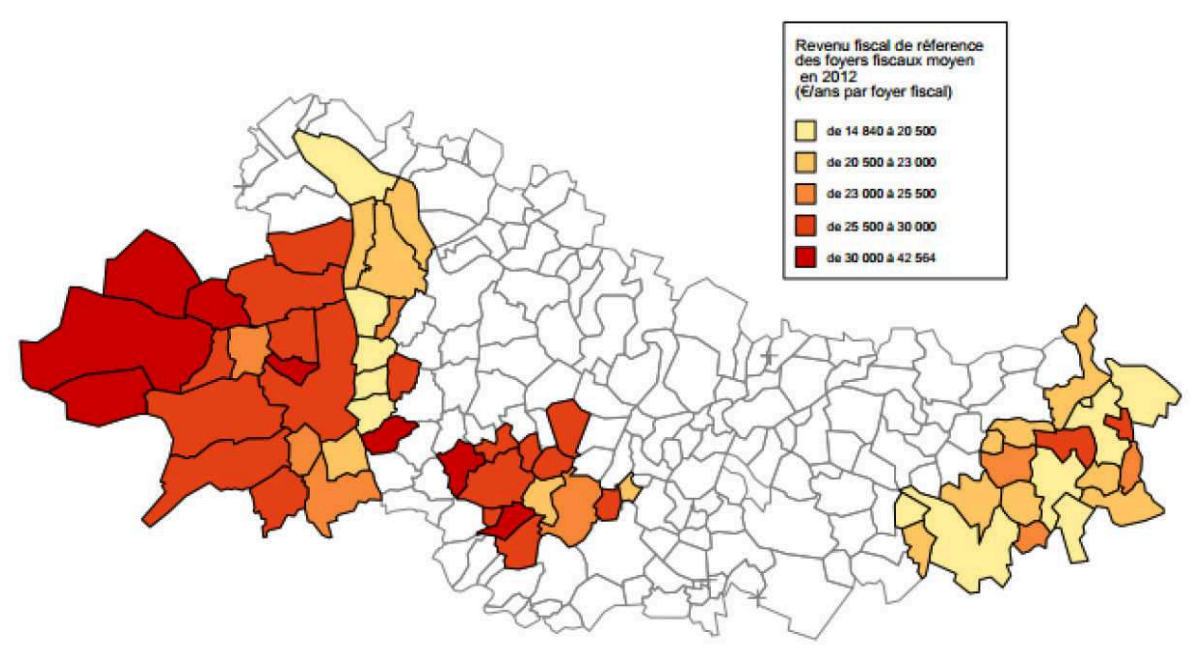

Ces différences territoriales nous permettent de sélectionner des perturbateurs potentiels et d'émettre l'hypothèse qu'ils sont perçus différemment en fonction des territoires. Ainsi huit perturbateurs ont été retenus dans notre étude (Tableau 1).

Tableau 1. Liste des huit perturbateurs identifiés

\begin{tabular}{|l|l|l|l|l|}
\hline \multirow{2}{*}{ Perturbateurs } & \multicolumn{4}{l|}{ Conséquences possibles } \\
\cline { 2 - 6 } & économiques & sanitaires & sociales & psychologiques \\
\hline Baisse de revenus & X & X & X & X \\
\hline Problèmes de santé & X & X & X & X \\
\hline Recompositions familiales & X & X & X & X \\
\hline Retraite & X & X & X & X \\
\hline Chômage & X & X & X & X \\
\hline Difficultés d'adaptation aux nouvelles \\
technologies
\end{tabular}

Les conséquences observées peuvent être liées par des relations de causes à effets entre perturbateurs : par exemple, le chômage peut entraîner une baisse de revenus et, si ce manque d'argent entraîne un déficit de soins, peut générer des problèmes de santé. Autre exemple: des recompositions familiales ou l'accès à la retraite peuvent aussi avoir des conséquences sanitaires suite à une baisse de revenus mais également suite à 
l'arrêt d'une prise en charge automatique (médecine du travail, mutuelle, etc.). Ces perturbateurs présentent-ils des différences entre les territoires ? Sont-ils associés à un sentiment de déclassement social?

\section{2) Liens entre perturbateurs, sentiment de déclassement social et territoires}

Les résultats à la question «Êtes-vous, ou pourriez-vous dans les 2 ans à venir, être concerné par les perturbateurs suivants? $»^{2}$ montrent que les répondants se disent significativement plus concernés par la baisse de revenus et les problèmes de santé que par les autres perturbateurs ${ }^{3}$. Cependant, aucune différence territoriale n'est observée pour ces deux perturbateurs. Cela s'explique par le fait que ces derniers peuvent concerner chaque personne, à tout moment de sa vie, quel que soit le territoire. À l'inverse, des différences territoriales sont observées pour trois autres perturbateurs : le chômage $(\mathrm{p}<0,001)$ et les recompositions familiales $(\mathrm{p}<0,01)$, dont les résultats varient dans le sens d'un gradient décroissant de la métropole bordelaise au Pays Foyen, et la retraite $(p<0,05)^{4}$ dont le gradient est inverse. Ainsi, sur les huit perturbateurs estimés pertinents pour cette étude, les deux jugés les plus préoccupants (une baisse de revenus et des problèmes de santé) ne varient pas en fonction des territoires car concernent tout le monde; trois présentent des différences territoriales (le chômage, les recompositions familiales et la retraite) en lien avec les disparités sociodémographiques mises en évidence par le diagnostic territorial; et les trois autres (devenir propriétaire de son logement; difficultés d'adaptation aux nouvelles technologies; évolution défavorable de son quartier) ne montrent aucune différence territoriale.

L'analyse des résultats concernant le sentiment de déclassement social indique une bonne répartition des répondants entre ceux qui perçoivent une dégradation de leur niveau de vie (score négatif pour les classes a et $b$ réunies, $n=146$ ) et ceux qui perçoivent une amélioration (score positif pour les classes $c$ et $d$ réunies, $\mathrm{n}=132$ ). La moyenne globalement associée au déclassement est négative : $x_{\text {moy }}=-0,35 \pm 0,12 \mathrm{~cm}$. Ainsi, environ la moitié des personnes interrogées (53\%) est considérée comme étant en situation de déclassement social (Figure 5). 


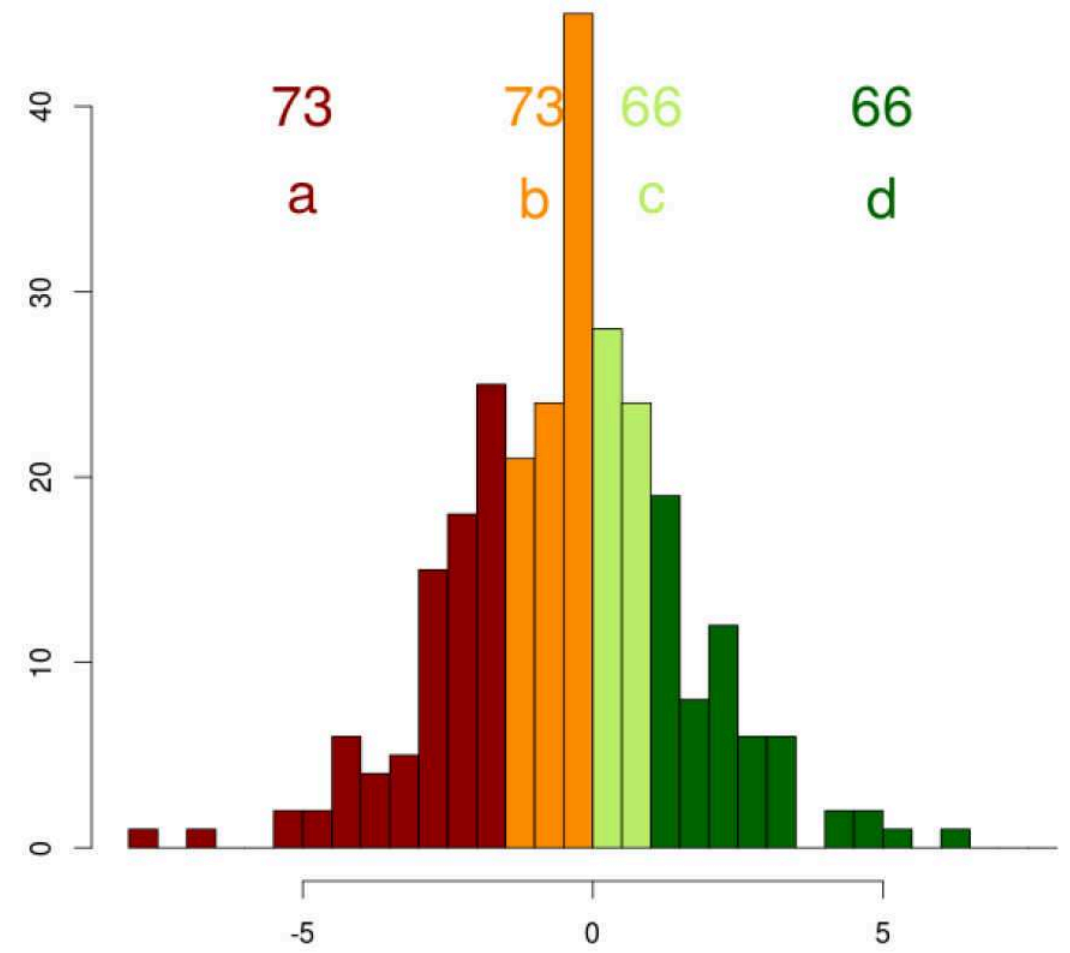

Bien que les pourcentages de personnes affectées dans chacune des 4 classes diffèrent en fonction des territoires, ces différences territoriales ne sont pas statistiquement significatives. De plus, aucune des variables socio-démographiques testées (sexe, âge, logement, niveau d'étude ou profession) n'est liée au sentiment de déclassement social ${ }^{5}$. Ces résultats renforcent l'hypothèse de l'existence d'une dimension subjective du déclassement, indépendante du niveau de ressources, statut de propriété, ou niveau d'étude, qui se traduit bien par un sentiment de déclassement. Cependant, un lien significatif est observé entre le sentiment de déclassement social et certains perturbateurs: quatre d'entre eux en effet sont vécus comme des causes du déclassement ressenti. Il s'agit de la baisse de revenus ( $p<0,0001)$, du chômage $(p<0,01)$, des recompositions familiales $(p<0,05)$ et des problèmes de santé $(p<0,05)^{6}$. Qu'en est-il de la pratique des comportements écologiques?

\section{3) Comportements écologiques et motivations identitaires}

Des différences territoriales sont mises en évidence pour un certain nombre de comportements écologiques: la pratique de l'ensemble de ces comportements, et plus spécifiquement l'autoproduction et la prolongation de la durée de vie des produits ${ }^{7}$. Ces différences, qui caractérisent un gradient croissant de mise en œuvre des pratiques de la métropole bordelaise au Pays Foyen, sont peu expliquées par les variables sociodémographiques mesurées (niveau d'étude ou profession; statut de propriété et type d'habitat - collectif / individuel; niveau de ressources) sauf par l'âge. Par contre, le déclassement social n'impacte pas directement ces comportements écologiques. L'adoption de ces comportements ne semble donc réservée ni à des personnes se sentant vulnérables et qui perçoivent une diminution de leur qualité de vie, ni à une frange aisée de la population qui a le luxe de pouvoir choisir son mode de 
consommation. S'il n'y a pas de différence de fréquence cela ne veut pas dire pour autant qu'il n'y en ait pas de nature. L'absence de lien pourrait en partie être expliquée par le fait que les cinq catégories de pratiques écologiques retenues correspondent à un panel de comportements variés (par exemple pour la mutualisation : co-voiturage ou échange d'outils), ne mobilisant pas les mêmes moyens, ni les mêmes motivations, et pouvant donc être adoptés par des personnes, voire des groupes sociaux, différents. Néanmoins, un lien positif et significatif est observé entre la pratique des comportements écologiques et la satisfaction des motivations identitaires ${ }^{8}$. En d'autres termes, plus les gens disent pratiquer des comportements écologiques, plus l'ensemble de leurs motivations identitaires sont satisfaites, et inversement. Cette corrélation est plus ou moins forte en fonction des comportements d'une part, et en fonction des motivations identitaires spécifiques d'autre part (Tableau 2).

Tableau 2. Relations entre comportements écologiques et motivations identitaires

\begin{tabular}{|c|c|c|c|c|c|c|}
\hline & \multicolumn{6}{|c|}{ Motivations identitaires } \\
\hline & Appartenance & Sens & Efficacité & \begin{tabular}{|ll} 
Estime de \\
Soi
\end{tabular} & Continuité & Distinctivité \\
\hline Autoproduction & $-0.04(a)$ & $0.18^{* *}(a b)$ & $0.12 *(a b)$ & $0.26^{* * *}(\mathrm{~b})$ & $0.25^{* * *}(\mathrm{~b})$ & $0.24^{* * *}(\mathrm{~b})$ \\
\hline Prolongation & $-0.04(a)$ & $0.28^{* * *}(\mathrm{c})$ & $0.29^{* * *}(\mathrm{c})$ & $0.23^{* * *}(\mathrm{bc})$ & $0.31^{* * *}(\mathrm{c})$ & $0.08(a b)$ \\
\hline Mutualisation & $0.09(\mathrm{a})$ & $\begin{array}{l}0.24^{* * *} \\
(\mathrm{ab})\end{array}$ & $\begin{array}{l}0.27^{* * *} \\
(\mathrm{ab})\end{array}$ & $0.22^{* * *}(\mathrm{a})$ & $0.39^{* * *}(\mathrm{~b})$ & $0.14^{*}(\mathrm{a})$ \\
\hline Réduction & $0.19^{* *}(\mathrm{a})$ & $0.21^{* *}(\mathrm{ab})$ & $0.18^{* *}(\mathrm{a})$ & $0.20^{* *}(\mathrm{ab})$ & $0.39^{* * *}(\mathrm{~b})$ & $0.27^{* * *}(\mathrm{ab})$ \\
\hline Substitution & $0.16^{*}$ & $0.17^{*}$ & $0.28^{* *}$ & $0.21^{* *}$ & $0.25^{* * *}$ & 0.09 \\
\hline
\end{tabular}

Dans le tableau 2, sont indiqués : les coefficients de corrélation de Spearman $(r)$ entre comportements écologiques et motivations, le niveau de significativité de la corrélation $(p<0,05: * ; p<0,01$ : **; $p<0,001: * \star \star)$. Les lettres différentes indiquent une différence significative entre motivations.

Il apparaît que tous les comportements écologiques sont fortement liés à la motivation de continuité (sentiment d'être la même personne quels que soient le lieu, la situation et le contexte). Globalement, toutes les pratiques écologiques confèrent aux personnes une bonne image d'elles-mêmes (estime de soi) mais plus particulièrement, autoproduire, prolonger la vie des produits et partager. Prolonger, partager et remplacer donnent à la personne le sentiment d'être compétente et capable (efficacité). Prolonger la durée de vie des produits et partager, autrement dit, ne pas gaspiller et tisser du lien social, confèrent du sens à la vie de la personne (sens). L'autoproduction permet de se sentir distinct des autres, d'exprimer sa personnalité au travers de la confection de vêtements, de meubles ou de la décoration par exemple (distinctivité). En raison des exemples proposés aux répondants dans le questionnaire (j'achète moins, je chauffe moins ma maison, je réduis l'usage de la climatisation), l'association de la réduction à la motivation de distinctivité peut être expliquée par le sentiment qu'ont les répondants de mettre en œuvre les écogestes concernant les économies d'énergie contrairement à la plupart des gens. On peut également penser qu'ils se sentent « plus 
malins » en mettant en œuvre des façons originales de réduire leurs consommations, ce qui peut les conduire à se percevoir comme uniques.

Ces résultats montrent clairement l'importance des liens entre identité et comportements écologiques et suggèrent que ces comportements peuvent représenter un support de valorisation sociale apportant un gain psychologique non négligeable en termes d'auto-évaluation personnelle.

\section{Conclusions}

28 Finalement, quels leviers d'action mobiliser à l'échelle territoriale pour atténuer la dégradation de la qualité de vie des personnes vulnérables, et réduire les préjudices induits par les restrictions et les modifications d'accès à certains postes de consommation de biens et services, tout en valorisant les comportements écologiques?

Nos résultats montrent d'une part que des différences territoriales sont observées pour trois perturbateurs (le chômage, les recompositions familiales et la retraite) et plusieurs types de comportements écologiques (la pratique de l'ensemble de ces comportements, l'autoproduction et la prolongation de la durée de vie des produits). D'autre part, environ la moitié des répondants se considèrent comme étant en situation de déclassement social. Ce sentiment de déclassement social est influencé par quatre perturbateurs (baisse de revenus, chômage, problèmes de santé et recompositions familiales) mais n'apparaît ni affecté par des critères territoriaux et sociodémographiques, ni ne semble influencer l'adoption des comportements écologiques. Cependant, la pratique des comportements écologiques a des conséquences positives sur la satisfaction des motivations identitaires des personnes, quel que soit leur sentiment de déclassement social. De ce fait, l'adoption de nouvelles valeurs peut amener les individus à percevoir comme positifs des comportements jusqu'alors envisagés comme contraignants, ce qui pourrait alors contribuer à un processus dynamique de résilience.

Les résultats de cette étude engagent donc à prendre en compte la dimension subjective $\mathrm{du}$ déclassement social et à développer des actions susceptibles de promouvoir l'adoption de comportements écologiques, en tant que stratégies adaptatives, auprès de l'ensemble des personnes qui estiment leur qualité de vie menacée. Il paraît tout d'abord important de mettre l'accent sur le caractère distinctif, en faisant le lien entre un comportement écologique et un renforcement de l'image positive de soi. En relation avec la motivation de continuité, l'adoption de nouveaux comportements doit s'inclure dans le style de vie des personnes et leur système de valeurs, et ne doit pas être vécue comme une rupture. Enfin, l'adoption de nouveaux comportements écologiques doit faciliter la montée en capacité ou l'empowerment, ce qui est particulièrement important pour les personnes se ressentant en déclassement, souvent dépendantes et qui ont donc davantage besoin de prouver qu'elles ont un sentiment de contrôle de leur existence. Du point de vue de l'efficacité des actions visant à promouvoir les comportements pro-environnementaux il est donc essentiel de mettre au premier plan les avantages pour l'individu en termes de valorisation sociale et personnelle et de transformer des choix dictés par la nécessité en choix de liberté.

31 Concernant les moyens d'accompagnement, les résultats du questionnaire ${ }^{9}$ indiquent que ce sont avant tout les conseils et l'information dispensés par les proches et les pairs 
qui pourraient aider les personnes à adopter des comportements écologiques. La promotion des comportements écologiques par les collectivités et les centres d'animation socio-culturelle, pourtant active, semble au contraire assez peu perçue par les répondants. Une communication plus efficace de ces structures au plus près de la diversité des personnes et de leurs modes de vie semble donc à privilégier. Enfin, ces actions doivent être en lien avec les spécificités territoriales : l'identité de lieu jouant au même titre que l'identité sociale, les comportements écologiques doivent s'inscrire dans un collectif spatialement circonscrit et pourvoyeur de lien social (comme une structure de quartier ou de voisinage). De plus, les enjeux écologiques lointains ne pouvant être assimilés et appropriés qu'au travers du filtre de la proximité, les leviers d'action doivent donc également s'appuyer sur les territoires vécus et leur hétérogénéité.

\section{BIBLIOGRAPHIE}

Alier J. M. (2014), L'écologisme des pauvres. Une étude des conflits environnementaux dans le monde, Paris, Les petits malins/Institut Veblen pour les réformes économiques.

Ballesta O., Carimentrand A., Causse E., Delerue F., Felonneau M. L., Gombert-Courvoisier S. et Ribeyre F. (2016), « L'adoption de comportement écologiques face au déclassement social : éléments préliminaires ", in Bourg D., Dartigupeyrou C., Gervais C. et Perrin O. (dir.), Les nouveaux modes de vie durables, s'engager autrement, Lormont, Le bord de l'eau, p. 107-112.

Boisson M. (2009), La mesure du déclassement, La documentation française, $\mathrm{n}^{\circ} 20$.

Bourg D., Dartigupeyrou C., Gervais C. et Perrin O. (2016), Les nouveaux modes de vie durables, s'engager autrement, Lormont, Le bord de l'eau.

Felonneau M. L. et Becker M. (2008), « Pro-environmental attitudes and behavior: revealing perceived social desirability », Revue Internationale de Psychologie Sociale, $n^{\circ}$ 21, p. 25-53.

Inove.com (2016), «Identité, NOrme, Vulnérabilité, pour une approche Écologique de la COnsommation durable des Ménages », Rapport de recherche, Programme MOVIDA, Convention $n^{\circ}$ 13-MUTS-MOVIDA-2-CVS-015, n Chorus 2101159 512, 29/10/03-29/04/16.

Laurent E. (2010), « Environmental justice and environmental inequalities: a European perspective ", hal-01069412, disponible sur : https://hal-sciencespo.archives-ouvertes.fr/ hal-01069412.

Maurin E. (2009), La peur du déclassement, une sociologie des récessions, Paris, Seuil.

Pye S., Skinner I., Meyer-Ohlendorf N., Leipprand A., Lucas K. et Salmons R. (2008), Addressing the social dimensions of environmental policy. A case study on the linkages between environmental and social sustainability in Europe, European Commission Directorate, General Employment, Social Affairs and Equal Opportunities.

Vignoles V., Regalia C., Manzi C., Golledge L. et Scabini E. (2006), « Beyond self-esteem: influence of multiple motives on identity construction ", Journal of Personality and Social Psychology, n 90 (2), p. 308-333. 


\section{NOTES}

1. Une baisse de revenus; des problèmes de santé; des recompositions familiales, une séparation... ; la retraite ; le chômage ; des difficultés d'adaptation aux nouvelles technologies ; devenir propriétaire de son logement ; une évolution défavorable de son quartier.

2. Scores de réponses variant de $1:$ pas du tout à $6:$ tout à fait.

3. Test de Friedman. Différence significative au seuil $\mathrm{p}<0,05$.

4. Test de Kruskall-Wallis. Relation significative au seuil $p<0,05$.

5. Test de corrélation de Spearman, $\mathrm{p}>0,05$.

6. ANOVA. Relation significative au seuil $\mathrm{p}<0,05$.

7. Test de Kruskall-Wallis. Relation significative au seuil $\mathrm{p}<0,05$.

8. Test de corrélation de Spearman. $r=0,46, p<0,001$.

9. Non montrés dans cet article.

\section{RÉSUMÉS}

L'étude porte sur trois territoires de Gironde (France), situés le long d'un gradient d'urbanisation : la métropole de Bordeaux, le territoire périurbain du Créonnais et le territoire rural du Pays Foyen. Un diagnostic cartographique comparatif a été effectué à partir d'indicateurs socio-démographiques et économiques empruntés aux bases de données publiques (INSEE) pour caractériser les vulnérabilités territoriales et faire émerger des perturbateurs potentiels de la qualité de vie des personnes. Une enquête par questionnaire a aussi été réalisée ( $\mathrm{n}=285$ répondants) pour $: \mathrm{i}$ ) identifier quels perturbateurs semblaient les plus problématiques en fonction des territoires, ii) mesurer le sentiment de déclassement social des répondants et vérifier s'il était en partie déterminé par les perturbateurs et perçu différemment en fonction des territoires, iii) caractériser les comportements écologiques pratiqués et vérifier si leur adoption contribuait à satisfaire les motivations identitaires. L'objectif de cette étude est de vérifier si des changements de comportements vers des pratiques plus écologiques peuvent représenter des supports d'estime personnelle et ainsi contribuer à atténuer le sentiment de déclassement social des personnes vulnérables qui les adoptent.

\section{INDEX}

Mots-clés : qualité de vie, comportements écologiques, motivations identitaires, déclassement, estime de soi

Keywords : quality of life, ecological behavior, identity motivations, downgrading, self-esteem

\section{AUTEURS}

\section{SANDRINE GOMBERT-COURVOISIER}

UMR 5319 PASSAGES 
OLIVIER BALLESTA

UMR 5319 PASSAGES

AURÉLIE CARIMENTRAND

UMR 5319 PASSAGES

FRANCIS RIBEYRE

UMR 5319 PASSAGES

\section{ELSA CAUSSE}

EA 7352 CHROME

FLORIAN DELERUE

EA 4592 Géoressources et Environnement

MARIE-LINE FÉLONNEAU

EA 4139 Laboratoire de Psychologie

GEOFFREY RIOCHE

EA 4139 Laboratoire de Psychologie 